\title{
Aggressive Grade 2 Neuroendocrine Tumor Salvaged by Temozolomide- Based Therapy: A Case Report and Literature Review \\ Ralph Chebib ${ }^{1}$, Roland Eid ${ }^{1}$, Fadi Farhat ${ }^{1}$, Joseph Kattan ${ }^{1 *}$ and Claude Ghorra ${ }^{2}$
}

${ }^{1}$ Department of Hematology-Oncology, Hôtel-Dieu de France University Hospital, Beirut, Lebanon

${ }^{2}$ Faculty of Medicine, Department of Pathology, Hôtel-Dieu de France University Hospital, Saint Joseph University, Beirut, Lebanon

\begin{abstract}
We are reporting a 59-year-old woman with a grade 2 (G2) moderately differentiated metastatic neuroendocrine (NET) tumor of unknown origin with Ki67 rate of $15 \%$. First-line treatment with etoposide and cisplatin failed with evidence of rapid disease progression. However, second-line therapy with temozolomide associated to capecitabine proved an unexpected efficacy resulting in a consistent partial response. Immunohistochemistry staining for O-6methylguanine-DNA methyltransferase (MGMT) expression was performed retrospectively and was negative, which could predict response to temozolomide. Systematic treatment strategies of non-well differentiated NETs are reviewed, as well as the role of MGMT as predictive factor for the efficacy of temozolomide.
\end{abstract}

Keywords: Neuroendocrine tumor; Moderately differentiated; Temozolomide; MGMT

\section{Introduction}

Computed Neuroendocrine tumors (NETs) have been recently classified by World Health Organization (WHO) criteria into welldifferentiated neuroendocrine tumors grade 1 (WD-NETs G1), welldifferentiated neuroendocrine tumors grade 2 (WD-NETs G2), and poorly differentiated neuroendocrine tumors (PD-NETs G3) [1]. G1 NETs classification derived from a mitotic count of less than $2 / 10$ highpower field (HPF) and/or a Ki-67 index of less than $3 \%$. In G3 NETs, the mitotic count usually exceeds 20/10 HPF and/or the Ki-67 index exceeds $20 \%$. While G2 represent the group of tumors that fall right between G1 and G3 tumors with a mitotic count ranging from 2 to 20/10 HPF and/ or Ki-67 that falls between the two extreme levels of $2 \%$ and $20 \%$.

Treatment guidelines based on those histological classifications have been recommended mainly for the grade 1 where somatostatine analogs are the mainstay therapy, and grade 3 tumors where the aggressive combination of platinum and etoposide is the standard of care. There is a lack of consensus on the optimal treatment strategy for grade 2 tumors, and only limited data from retrospective studies is available in the literature.

We report a case of highly aggressive and multi-metastatic NET disease classified as G2 tumor, where resistance to the combination of etoposide and cisplatinum was encountered contrasting with a sensitivity to temozolomide-based therapy.

\section{Case Report}

A 59-year-old female presented to the office complaining of atypical back pain. The pain started a few months ago, increasing gradually in intensity, along with loss of appetite and $10 \%$ loss of her weight. The spine MRI showed multiple lytic bony lesions involving the dorsal and lumbo-sacral spine. Total body scan revealed multiple subcentimeter pulmonary nodules as well as multiple secondary hepatic lesions and bilateral adrenal suspicious enlargement. The patient underwent a transcutaneous biopsy of the adrenal gland. Histomorphological examination revealed monomorphic cell nuclei and lack of necrosis. Ten mitosis per $10 \mathrm{HPF}$ were counted and Ki67 was $15 \%$. On immunohistochemistry (IHC), the tumor was highly positive for synaptophysine and chromogranine receptor, moderately positive for $\mathrm{CK} 7$, low positive for TTF-1, and negative for CK20. The diagnosis of a moderately differentiated (G2) neuroendocrine tumor was confirmed. Gallium scan showed the same pattern of disease extension but failed to identify a primary lesion. Chromogranin serum assessment was not done. 24hour urine 5-hydroxyindoleacetic acid was within normal range. The patient received cytotoxic chemotherapy with etoposide $100 \mathrm{mg} / \mathrm{m}^{2}$ intravenously (IV) on days 1 to 3 and cisplatin $75 \mathrm{mg} / \mathrm{m}^{2}$ day 1 of a 21 day cycle for four cycles, associated with zoledronic acid $4 \mathrm{mg}$ IV. Pain and weight loss increased after the second cycle, along with progression of the lesions by more than $75 \%$ on subsequent disease assessment. She received second-line therapy with temozolomide $150 \mathrm{mg} / \mathrm{m}^{2}$ divided into two doses daily on days 1 to 5 , and capecitabine $600 \mathrm{mg} / \mathrm{m}^{2}$ orally twice daily on days 1-14 of a 21-day cycle. After the completion of her second cycle, the patient presented with a complete disappearance of her pain, regain of appetite and weight, as well as a decrease in disease extension of more than $30 \%$. Actually, the disease remains in sustained partial response while the patient is receiving the eighth cycle. MGMT IHC was subsequently performed (Caris Life Sciences) and was negative (2\% staining).

\section{Discussion}

Despite the heterogeneity of NETs, the diagnosis confirmation relies on IHC that reveals positivity for neuroendocrine markers such as synaptophysin and chromogranin. They may also be associated with specific symptoms related to peptide release, which differentiates functioning from nonfunctioning tumors.

The recommended treatment for PD-NETs G3 is chemotherapy using etoposide (VP 16) and cisplatin (CDDP) [2]. This treatment strategy is extrapolated from the efficacy of this regimen in extensive

*Corresponding author: Dr. Joseph Kattan, Department of Hematology-Oncology Hôtel-Dieu de France University Hospital, Bvd A. Naccache - Achrafieh, Postal code-166830, Lebanon, Tel: +9613635913; E-mail: jkattan62@hotmail.com; joseph. kattan.2018@gmail.com

Received: July 11, 2018; Accepted: July 31, 2018; Published: August 03, 2018

Citation: Chebib R, Eid R, Farhat F, Kattan J, Ghorra C (2018) Aggressive Grade 2 Neuroendocrine Tumor Salvaged by Temozolomide-Based Therapy: A Case Report and Literature Review. Oncol Cancer Case Rep 4: 147.

Copyright: @ 2018 Chebib R, et al. This is an open-access article distributed under the terms of the Creative Commons Attribution License, which permits unrestricted use, distribution, and reproduction in any medium, provided the original author and source are credited. 
stage small cell lung cancer since 1991. At that time, Moertel et al. proved its efficacy in treating anaplastic neuroendocrine carcinomas of the lungs with an objective tumor regression of $67 \%$, median progression free survival (PFS) of 9 months and median overall survival (OS) of 19 months [3]. In a subsequent study of 36 patients with advanced NETs (either poorly-differentiated histology or a rapidly progressing clinical course), second-or even third-line with cytotoxic chemotherapy cisplatin and etoposide yielded an overall radiologic response rate of $36 \%$ and a median OS of 19 months [4]. Furthermore, and with the same regimen, Mitry et al. reported a similar response rate (42\%) and median OS of 15 months in a retrospective analysis of 41 patients with poorly differentiated NETs. Interestingly, only 1 of 11 patients with welldifferentiated NET responded [5]. This was further analyzed in a recent retrospective study: The NORDIC NEC study, where 305 patients with advanced gastro-intestinal NETs were included. The authors concluded that patients with $\mathrm{Ki}-67<55 \%$ were less responsive to platinum-based chemotherapy but had longer survival [6].

In contrast to poorly differentiated NETs, the advanced or metastatic well-differentiated, slow growing $(\mathrm{Ki}-67<2 \%)$ NETs are treated with somatostatine analogs. The PROMID study, showed a statistically significant median PFS benefit of 14.3 months versus 6 months in favor of octreotide LAR [7]. Lanreotide, in the CLARINET study, was also associated with a significantly prolonged PFS with median not reached versus median of 18.0 months in the placebo arm [8]. However, there is a lack of effective cytoreductive regimens without substantial toxicity. Historically, streptozotocin (STZ), STZ \pm doxorubicin or 5-fluorouracil (5-FU) were the only approved regimens for metastatic pancreatic NETs (PNETs). However, older studies were mostly retrospective, and the fact that they often lacked the use of strict radiographic criteria for response evaluation accounts for their low credibility. When RECIST indices were accurately utilized in recent studies, response rates ranged from 6 to $16 \%$, and substantial grade 3/4 toxicities were observed [9].

Dacarbazine (DTIC), a member of the class of alkylating agents, was prospectively studied for metastatic PNETs as a high-dose single agent with an ORR of 34\% [10]. Temozolomide (TMZ), which is an oral formulation of the first metabolite of DTIC has a more favorable toxicity profile. In the earliest reports in 2006, TMZ, in a phase 2 study, in combination with thalidomide produced a combined RR of $25 \%$ (45\% in PNETs, $33 \%$ in pheochromocytomas and $7 \%$ in carcinoids) and a median PFS of 13.5 months. The major downside was significant toxicity where $69 \%$ of patients developed grade 3-4 lymphopenia, and $10 \%$ had opportunistic infections [11].

While single agent capecitabine did not succeed in providing a substantial activity in a small phase 2 study [12], its association with temozolomide proved to be an exciting combination. As a basic rationale, capecitabine induces DNA damage by incorporation of 5-FdUTP into DNA resulting in an inhibition of thymidylate synthase via 5-FdUMP and reducing thymidine pools. Thus, the addition of temozolomide and its effect on O6-alkylguanyl alkyl-transferase (O6-AGAT) is synergistically enhanced by capecitabine [13], which acts by depleting the DNA repair enzyme O6 methylguanine DNA methyltransferase (MGMT) making it more sensitive to the antiproliferative effects of temozolomide [14]. Kulke et al. published a retrospective analysis on MGMT expression in PNETs and carcinoid tumors. MGMT was found to be deficient in $50 \%$ of PNETs vs. $0 \%$ of carcinoids tumors, explaining the decreased tumor response to temozolomide in the latter [11]. This theoretical inefficacy was questioned by the fact that temozolomide and capecitabine combination, given in two daily dosages as proposed by Fine et al. in their pilot study of this regimen, resulted in atol response rate of $61 \%$, and a clinical benefit of $83.2 \%$ [15]. More recently, and using the same combination, an interim analysis of a phase II trial reported a response rate of $42 \%$, including $8 \%$ CR and $58 \%$ SD with a median PFS of 22 months in patients with metastatic, well-to-moderately differentiated neuroendocrine tumors who had disease progression on long-acting octreotide (Sandostatin LAR), $60 \mathrm{mg}$, or who had negative octreotide scans, which is a negative prognostic factor [16].

\section{Conclusion}

At presentation, our patient with rapidly progressing disease behaved more like a G3 than a G2 disease, despite a Ki-67 of less than $20 \%$. The combination of etoposide and cisplatin was thought to be the adequate strategy. However, the disease failed to respond and even progressed after three cycles. There was a strong unexpected partial response to second-line temozolomide and capecitabine. We subsequently performed immunostaining with anti-MGMT antibody on our patient's adrenal gland paraffin sections. The markedly low pattern of MGMT expression provides a clear explanation for the sensitivity of our patient's tumor to temozolomide. This finding, when correlated with Fine et al.s hypothesis on MGMT depletion with the BID administration of temozolomide, might prove to be an important piece of the puzzle in order to determine the best first-line treatment to propose in patients with WD-NETs G2 tumors.

\section{Acknowledgments}

We are thankful to the patient who accepted to publish her data in the text. A consent has been obtained from the patient, to publish her data in this case report.

\section{References}

1. Bosman FT, Carneiro F, Hruban RH, Theise ND (2010) WHO classification of tumours of the digestive system. [Internet]. World Health Organization, Geneva.

2. Arnold R (2005) Introduction: Definition, historical aspects, classification staging, prognosis and therapeutic options. Best Pract Res Clin Gastroenterol 19: 491-505.

3. Moertel CG, Kvols LK, O'Connell MJ, Rubin J (1991) Treatment of neuroendocrine carcinomas with combined etoposide and cisplatin. Cancer 68 227-232.

4. Fiällskog ML, Granberg DP, Welin SL, Eriksson C, Oberg KE, et al. (2001) Treatment with cisplatin and etoposide in patients with neuroendocrine tumors. Cancer 92: 1101-1107.

5. Mitry E, Baudin E, Ducreux M, Sabourin JC, Rufié P, et al. (1999) Treatment of poorly differentiated neuroendocrine tumours with etoposide and cisplatin. $\mathrm{Br}$ Cancer 81: 1351-1355.

6. Sorbye H, Welin S, Langer SW, Vestermark LW, Holt N, et al. (2012) Predictive and prognostic factors for treatment and survival in 305 patients with advanced gastrointestinal poorly differentiated neuroendocrine carcinoma: The Nordic NEC study. Ann Oncol 24: 152-160.

7. Rinke A, Müller HH, Schade-Brittinger C, Klose KJ, Barth P, et al. (2009) Placebo-controlled, double-blind, prospective, randomized study on the effect of octreotide LAR in the control of tumor growth in patients with metastatic neuroendocrine midgut tumors: a report from the PROMID Study Group. J Clin Oncol 27: 4656-4663.

8. Caplin ME, Pavel M, Ćwikła JB, Phan AT, Raderer M, et al. (2014) Lanreotide in metastatic enteropancreatic neuroendocrine tumors. N Engl J Med 371: 224233

9. Sun W, Lipsitz S, Catalano P, Mailliard JA, Haller DG (2005) Phase II/III study of doxorubicin with fluorouracil compared with streptozocin with fluorouracil or dacarbazine in the treatment of advanced carcinoid tumors: Eastern Cooperative Oncology Group Study E1281. J Clin Oncol 23: 4897-4904.

10. Ramanathan RK, Cnaan A, Hahn RG, Carbone PP, Haller DG (2001) Phase I trial of dacarbazine (DTIC) in advanced pancreatic islet cell carcinoma. Study of the Eastern Cooperative Oncology Group-E6282. Ann Oncol 12: 1139-1143.

11. Kulke $\mathrm{MH}$, Hornick JL, Frauenhoffer $\mathrm{C}$, Hooshmand S, Ryan DP, et al. (2009) $\mathrm{O}_{6}$-methylguanine DNA methyltransferase deficiency and response 
Citation: Chebib R, Eid R, Farhat F, Kattan J, Ghorra C (2018) Aggressive Grade 2 Neuroendocrine Tumor Salvaged by Temozolomide-Based Therapy: A Case Report and Literature Review. Oncol Cancer Case Rep 4: 147.

Page 3 of 3

to temozolomide-based therapy in patients with neuroendocrine tumors. Clin Cancer Res 15: 338-345

12. Medley L, Morel AN, Farrugia D, Reed N, Hayward N, et al. (2011) Phase II study of single agent capecitabine in the treatment of metastatic non-pancreatic neuroendocrine tumours. Br J Cancer 104: 1067-1070.

13. Fine RL, Fogelman DR, Schreibman SM (2005) Effective treatment of neuroendocrine tumors with temozolomide and capecitabine. Journal of Clinical Oncology p: 4216.

14. Murakami J, Lee YJ, Kokeguchi S, Tsujigiwa H, Asaumi JI, et al. (2007) Depletion of $\mathrm{O}_{6}$-methylguanine-DNA methyltransferase by $\mathrm{O}_{6}$-benzylguanine enhances 5-FU cytotoxicity in colon and oral cancer cell lines. Oncol Rep 17 1461-1467.

15. Fine RL, Gulati AP, Krantz BA, Moss RA, Schreibman S, et al. (2013) Capecitabine and temozolomide (CAPTEM) for metastatic, well-differentiated neuroendocrine cancers: The Pancreas Center at Columbia University experience. Cancer Chemother Pharmacol 71: 663-670.

16. Fine RL, Gulati AP, Tsushima D, Mowatt KB, Oprescu A, et al. (2014) Prospective phase II study of capecitabine and temozolomide (CAPTEM) fo progressive, moderately, and well-differentiated metastatic neuroendocrine tumors. Journal of Clinical Oncology p: 179 